\title{
Asociación entre los patrones alimentarios durante el primer año de vida y alergia alimentaria en lactantes
}

\author{
Association of dietary patterns and food allergy in infants \\ during the first year of life
}

\author{
Dra. Karla Amada Bascuñán Gamboa ${ }^{a}$, Dr. Sergio Gerardo Weisstaub Nuta , \\ Dr. Rodrigo Andrés Chamorro Melo , Dra. María Antonieta Guzmán ${ }^{c} y$ \\ Dra. Magdalena Araya Quezada ${ }^{b}$
}

\section{RESUMEN}

Introducción. Existe controversia acerca del efecto del patrón alimentario durante el primer año de vida y el desarrollo de alergia alimentaria. El objetivo de este estudio fue evaluar la asociación entre antecedentes familiares de alergia, manifestaciones alérgicas y patrones alimentarios del primer año de vida en lactantes con alergia alimentaria y sin ella.

Población y métodos. Se realizó un estudio descriptivo transversal en menores de 2 años $(n=99)$, distribuidos en dos grupos: alérgico $(n=50)$ y grupo control $(n=49)$, pareados por nivel socioeconómico, edad y género. Se definió alergia alimentaria según criterios clínicos internacionalmente aceptados, pruebas cutáneas y de parche, y respuesta a la dieta. Se recolectó información dietaria, clínica y de historia de alergia en los padres. Se calculó tamaño muestral para regresión logística (Freeman) y se utilizaron pruebas de Student, $\chi^{2}$ y Mann-Withney. El estudio y el consentimiento fueron aprobados por el Comité de Ética del INTA y de la Universidad de Chile. Resultados. El grupo alérgico mostró una prevalencia significativamente mayor $(p<0,0001)$ de historia familiar de alergia ( $84 \%$ ) que el grupo control $(16 \%)$. La diarrea fue la sintomatología más frecuentemente comunicada por las madres de los niños alérgicos durante el primer año de vida. La lactancia artificial se introdujo más tempranamente en el grupo alérgico que en el grupo control [ 3 contra 6 meses $(p<0,03)$ ]; no hallamos diferencias con respecto a la edad de inicio de la alimentación complementaria. Al realizar la regresión logística, solo la historia familiar de alergia se asoció con un mayor riesgo de presentar alergia alimentaria (OR: 48,2; IC $=14,2-164 ; \mathrm{p}<0,001$ ).

Conclusiones. La introducción precoz de formula láctea podría favorecer la presencia de alergia alimentaria en lactantes que presentan frecuentemente antecedentes familiares de alergia.

Palabras clave: alergia alimentaria, lactancia materna, alimentación complementaria, historia familiar, lactantes.

Dra. Magdalena Araya: maraya@inta.uchile.cl

Conflicto de intereses:

Ninguno que declarar.

Recibido: 14-11-2011

Aceptado: 27-4-2012

\section{INTRODUCCIÓN}

La alergia alimentaria se entiende como una falla del fenómeno de tolerancia oral en individuos predispuestos genéticamente, reacción que puede ser mediada por anticuerpos (IgE, IgG, IgA o IgM) o por células, con una amplia variedad de manifestaciones clínicas. ${ }^{1,2}$ La predisposición genética es un factor de riesgo importante, habiéndose descrito cifras de hasta el $70 \%$ de alergia en la descendencia de padres alérgicos. ${ }^{3}$ La base genética no puede ser modificada, ${ }^{4}$ pero existen factores ambientales que modulan la aparición de la alergia alimentaria; ${ }^{5}$ en este contexto, el rol de la dieta durante el primer año de vida podría ser determinante. $^{6}$

Las alergias alimentarias son más frecuentes en lactantes, presumiblemente por la inmadurez de mecanismos inmunológicos y no inmunológicos que previenen el ingreso de antígenos a través de la barrera gastrointestinal. ${ }^{78}$ Leche, huevo y maní son los alérgenos alimentarios más comunes en la temprana infancia y en la niñez. La alergia al maní, aunque menos común que la alergia al huevo o a la leche, más a menudo se asocia con reacciones alérgicas graves, incluida la anafilaxia. ${ }^{9}$

Estudios recientes indican que la prevalencia de alergia alimentaria ha aumentado en EE.UU. y Europa. ${ }^{10,11}$ Actualmente, este cuadro afecta aproximadamente al $2 \%$ de la población general y a cerca del 4-8\% de los niños estadounidenses. ${ }^{12}$ De acuerdo con un metaanálisis realizado predo- 
minantemente en países europeos, la prevalencia estimada para alergia alimentaria mediada por IgE oscila en $0-3 \%$ para la leche, $0-1,7 \%$ para el huevo y $0,2-1,6 \%$ para el maní. ${ }^{13}$

Recientemente, la Academia Estadounidense de Pediatría ${ }^{14}$ y la Sociedad Europea de Gastroenterología Pediátrica, Hepatología y Nutrición, ${ }^{15}$ publicaron sus nuevas guías sobre nutrición y prevención de alergia en lactantes y niños. Además de recomendar la lactancia materna exclusiva hasta los 6 meses, se propone que la alimentación complementaria no debería ser introducida antes de las 17 semanas y que todos los lactantes deberían comenzar la alimentación sólida no más allá de la semana $26 .{ }^{15}$ No se recomienda evitar o retardar la introducción de alimentos potencialmente alergénicos, como pescado y huevo. Además, no se recomienda una dieta de eliminación durante el embarazo y la lactancia. ${ }^{16}$

Las prácticas alimentarias representan un factor de riesgo potencialmente modificable para la prevención de la alergia. ${ }^{17}$ Factores relacionados con la dieta materna e infantil, como transferencia placentaria, leche materna, fórmulas infantiles y alimentación complementaria, representan la vía usual a través de la cual los lactantes entrarían en contacto con antígenos. La posibilidad de evitar la incorporación de alérgenos alimentarios a través de la manipulación de la dieta durante el embarazo o en la infancia temprana sigue siendo polémica. ${ }^{16}$

El objetivo de este estudio fue evaluar la asociación entre los patrones alimentarios durante el primer año de vida, la historia familiar de alergia y el riesgo de desarrollar alergia alimentaria en una muestra de lactantes chilenos menores de dos años.

\section{POBLACIÓN Y MÉTODOS}

La muestra estudiada provino de lactantes derivados a la sección de Inmunología, VIH y Alergias del Departamento de Medicina, del Hospital Clínico de la Universidad de Chile ( $\mathrm{HCUCH})$, que conformaron el grupo alérgico. La muestra de lactantes controles fue obtenida a partir de Centros de Salud y Guarderías de Santiago, los cuales fueron pareados por edad (intervalo de 15 días), sexo y nivel socioeconómico. Ambos grupos no fueron diferentes en términos de su etnia, pues se trabajó en la comuna de Santiago y no se incluyó a lactante alguno con padres de origen mapuche (principal grupo étnico nacional).

Criterios de inclusión: lactantes de 6-24 meses de edad con peso de nacimiento mayor a $2500 \mathrm{~g}$.

Criterios de exclusión: presencia de patologías como enfermedades genéticas, autoinmunitarias y metabólicas.

Criterios de eliminación: presencia de patología durante el período de estudio o bien que los padres rechazaran participar después del ingreso al protocolo.

Recolección de la información: Se realizó una entrevista semiestructurada, donde se aplicó un formulario ad-hoc a la madre. Se registraron los antecedentes clínicos, las pruebas cutáneas realizadas y la información que permitía confirmar el diagnóstico mediante la desaparición de los síntomas después de la dieta de eliminación.

Nivel socioeconómico (NSE): se tipificó a través de la clasificación propuesta por Esomar Chile. Este método, originario de Europa ha sido validado previamente en Chile; se basa en dos variables: ${ }^{18}$ el nivel de educación y la categoría ocupacional del principal sostenedor del hogar. Ambas variables se combinan en una "Matriz de clasificación socio-económica", que determina el NSE familiar de acuerdo con las combinaciones entre ambas variables.

Como variable dependiente se usaron las manifestaciones alérgicas (ver más adelante).

Las variables independientes fueron: i) historia familiar [historia familiar de alergia, presencia $\mathrm{o}$ ausencia de historia de alergia en el padre y/o madre y/o hermanos(as)]; ii) patrón alimentario (descrito según duración de lactancia materna y edad de inicio de alimentación complementaria, categorizadas en lactancia materna $\geq 4 \mathrm{~m}$ y alimentación complementaria $\geq 6 \mathrm{~m}$; lactancia materna $\geq 4$ m y alimentación complementaria $<6 \mathrm{~m}$; lactancia materna $<4 \mathrm{~m}$ y alimentación complementaria $\geq 6 \mathrm{~m}$; lactancia materna $<4 \mathrm{~m}$ y alimentación complementaria $<6 \mathrm{~m}$ ); iii) interacción de las variables.

Estado nutricional: tomando como base la norma técnica ministerial de evaluación del estado nutricional en menores de 6 años (instrumento que tiene como referente los estándares de crecimiento de la Organización Mundial de la Salud [OMS] 2007) se evaluó el estado nutricional a partir del indicador peso para la talla, mediante peso para la edad y talla para la edad. ${ }^{19}$

\section{Definiciones operacionales}

Diagnóstico de alergia en los niños: se basó en: i) las manifestaciones alérgicas descritas por el médico de atención primaria de salud que envió la interconsulta al Servicio de Inmunología, desde donde se recogieron los casos; ii) el resultado positivo de las pruebas cutáneas y las pruebas 
del parche a ciertos alimentos; y, iii) la mejoría de los síntomas luego de eliminado el alérgeno.

El diagnóstico clínico de alergia requirió el cumplimiento de los tres criterios arriba descritos y fue realizado en los lactantes que conforman el grupo alérgico. Los lactantes del grupo control se reclutaron en centros de salud primaria y salas cunas y jardines infantiles de la misma área geográfica (Región Metropolitana); en los controles la información se obtuvo de la ficha del niño, del carnet de controles de salud y mediante la encuesta aplicada durante la entrevista semiestructurada.

Manifestaciones alérgicas: los síntomas que motivaron la consulta inicial al médico hicieron que éste hiciera un diagnóstico presuntivo de alergia y derivara al paciente al especialista. Los síntomas se definieron operacionalmente: 1) piel: prurito recurrente, atopia, exantema o ronchas, eczema, urticaria; 2) respiratorio: sibilancias, signos obstructivos de más de $24 \mathrm{~h}$ que responden a broncodilatadores, rinitis alérgica; 3 ) digestivos: vómitos; diarreas; sangrado rectal; reflujo esofágico moderado-grave o constipación sin causa demostrada; dolor cólico que hace que el niño llore más de una hora por día, dos o tres veces por semana; 4) Otros: que el niño duerma menos de $6 \mathrm{~h}$ al día (se ha descrito mayor insomnio y fragmentación del sueño en lactantes alérgicos). ${ }^{20}$

Historia familiar: se consideró positiva cuando al menos un padre/madre y/o hermano tenía antecedentes de asma: cuando el diagnóstico de asma alérgica fue efectuado en un servicio hospitalario y/o el familiar utilizaba broncodilatadores.

- Rinoconjuntivitis: historia de uno o más de los siguientes síntomas: estornudos en salvas, obstrucción y prurito nasales y secreción mucosa transparente, acompañados de enrojecimiento ocular, prurito y lagrimeo, con edema palpebral.

- Eczema atópico: diagnóstico hecho por un médico, que incluyera dermatitis pruriginosa crónica o con recaídas crónicas; o si posee un historial de eczema con la típica aparición y localización (diagnóstico realizado por los especialistas tratantes).

- Alergia alimentaria: historia de prurito en la piel y/o urticaria y/o inflamación de los labios y/o dolor en los labios y lengua edematosa y/o vómitos y/o diarrea después de ingerir cierto tipo de alimentos, cumpliendo con tener una contraprueba positiva (reacción frente a la ingestión del antígeno alimentario).
Desde el punto de vista de la alimentación las definiciones operacionales fueron:

Patrón alimentario: la secuencia y tiempos de introducción de alimentos al lactante, incluyendo la presencia, duración de lactancia materna o artificial, y la fecha de inclusión de la alimentación complementaria y sus componentes.

Lactancia materna exclusiva: administración de leche materna como único alimento para cubrir las necesidades nutricionales, sin requerir otros alimentos o agregados. ${ }^{21}$

Alimentación complementaria: administración de cualquier alimento distinto a la leche. ${ }^{21}$

Consideraciones éticas. Previo a la entrevista, la madre y/o padre recibieron información detallada acerca del estudio y aquellos que accedieron a participar firmaron el consentimiento informado. El estudio y el consentimiento informado fueron evaluados y aprobados en marzo del año 2007 por el Comité de Ética para la investigación en seres humanos del INTA y del HCUCH.

Tamaño muestral y análisis estadístico. El tamaño muestral se calculó según el criterio de Freeman y cols. ${ }^{22}$ que propone diez eventos de interés por variable analizada; considerando las variables historia familiar de alergia, patrón alimentario, su interacción y manifestación alérgica, se calculó una muestra de 50 niños en cada grupo. Los datos fueron validados y se evaluó su normalidad mediante la prueba de Shapiro-Wilk. Se utilizó la prueba t de Student, ji al cuadrado y Mann-Withney para evaluar diferencias entre los grupos según su normalidad y el tipo de variable. Posteriormente, para evaluar el riesgo de alergia se utilizó un modelo de regresión logística que incluyó 3 variables independientes: historia familiar (2 categorías), patrón alimentario (4 categorías) e interacción, siendo la variable dependiente la manifestación alérgica (grupo alérgico/control). Se utilizó un grado de significación con un valor $\mathrm{p}<0,05$ y el análisis de los datos se realizó mediante el programa SPSS ${ }^{\circledast}$ v.15.0.

\section{RESULTADOS}

Se incluyeron en este estudio un total de 100 niños, 50 casos y 50 controles consecutivos, recolectados entre mayo 2007 y febrero 2008. La muestra final analizada quedó formada por 99 lactantes, 50 alérgicos y 49 controles sanos; se debió eliminar del estudio a un lactante del grupo control cuya madre decidió retirarse del estudio. Las características de los lactantes aparecen en la Tabla 1.

La diarrea fue la sintomatología más frecuentemente comunicada por las madres de los niños 
alérgicos durante el primer año de vida (Figura 1). La frecuencia de síndrome bronquial obstructivo no difirió entre ambos grupos.

En el grupo alérgico, el antecedente de historia familiar de alergia fue significativamente más frecuente $(84 \%$ contra $16 \%$ en controles, $p<0,001)$ $\mathrm{y}$ el indicador Talla/Edad actual fue significativamente menor $(71 \pm 7,2$ contra $74,3 \pm 7 \mathrm{~cm})$ en comparación con el grupo control ( $\mathrm{p}<0,03)$.

No hallamos diferencias entre los patrones alimentarios de ambos grupos. Casi la totalidad de los niños $(96 \%)$ recibió lactancia materna y la mediana (intervalo interquartil) de duración de la lactancia materna exclusiva fue de 4 (2-6) y 5 (2,5-6) meses en el grupo alérgico y control, respectivamente, sin diferencias significativas. Similarmente, la mediana (intervalo interquartil) de edad de inicio de la alimentación complementaria fue de 6 (5-6) meses en ambos grupos. Por el contrario, la introducción de la lactancia artificial ocurrió más tempranamente en el grupo alérgico: $3(1,4-16)$ contra $6(2,75-12)$ meses, respectivamente, $\mathrm{p}<0,03)$ al igual que la inclusión de alimentos distintos a la leche antes de los 6 meses de edad, siendo más temprana en el grupo alérgico $(p<0,02)$. Finalmente, no hubo diferencias en el tiempo de inclusión de cereales, pescados/mariscos, semillas (maní, nueces o almendras), carnes (rojas o blancas) o legumbres durante el primer año de vida.
El análisis de regresión logística univariada reveló que la historia familiar de alergia (OR: 48,29, [IC: 14,22- 164,04], p <0,001) -y no el patrón alimentario- determinó el mayor riesgo para presentar manifestaciones alérgicas. Al categorizar la variable duración de lactancia materna exclusiva ( $\leq 4$ meses o $>4$ meses) y la alimentación complementaria ( $<6$ meses $0 \geq 6$ meses) no hallamos diferencias significativas entre los grupos.

\section{DISCUSIÓN}

El presente estudio muestra que la presencia de manifestaciones alérgicas en menores de dos años se asocia principalmente a la historia familiar de alergia y a la incorporación precoz de formulas lácteas. La frecuencia de alergia en lactantes con antecedentes familiares de alergia en nuestra muestra ( $84 \%$ ) es mayor a lo comunicado para la heredabilidad de la condición atópica, ya que algunos informes indican que un $66 \%$ de los recién nacidos con antecedentes de atopia desarrollan la enfermedad alérgica. ${ }^{23,24}$

Nuestros resultados muestran que los lactantes con alergia alimentaria introdujeron más tempranamente la lactancia artificial en su alimentación, sin diferencias en la edad de introducción o duración de la lactancia materna. Lo primero concuerda con estudios que han demostrado que la introducción temprana de leche de

TABLA1. Características generales de la muestra

\begin{tabular}{|c|c|c|c|}
\hline \multirow[t]{2}{*}{ Variable } & \multicolumn{2}{|c|}{ Grupo } & \multirow[b]{2}{*}{ valor $\mathrm{p}$} \\
\hline & Alérgico $(n=50)$ & Control $(n=49)$ & \\
\hline Edad (meses) ${ }^{* *}$ & $12,4 \pm 5,2$ & $12,5 \pm 5,6$ & NS \\
\hline Sexo masculino (\%) & $26(52)$ & $24(49)$ & NS \\
\hline \multicolumn{4}{|l|}{ Nivel socioeconómico (\%) } \\
\hline Muy alto/alto & 32 & 31 & NS \\
\hline Medio-alto/medio & 56 & 45 & NS \\
\hline Medio-bajo/bajo & 12 & 24 & NS \\
\hline Presencia de antecedente familiar de alergia (\%) & 84 & 16 & $<0,001$ \\
\hline Peso de nacimiento $(\mathrm{g})^{* *}$ & $3318 \pm 435$ & $3386 \pm 420$ & NS \\
\hline Talla de nacimiento $(\mathrm{cm})^{* *}$ & $49,9 \pm 2,8$ & $50,1 \pm 1,8$ & NS \\
\hline \multicolumn{4}{|l|}{ Peso al nacimiento (\%) } \\
\hline 2500 a $2999 \mathrm{~g}$ & 30 & 22,4 & NS \\
\hline 3000 a $4000 \mathrm{~g}$ & 66 & 69,4 & NS \\
\hline$>4000 \mathrm{~g}$ & 4 & 8,2 & NS \\
\hline Peso actual $(\mathrm{g})^{*}$ & 8460 & 9840 & NS \\
\hline Talla actual $(\mathrm{cm})^{* *}$ & $71 \pm 7,2$ & $74,3 \pm 7$ & 0,03 \\
\hline
\end{tabular}

*Datos expresados como mediana; ${ }^{* *}$ datos expresados como promedio \pm desviación estándar.

Diferencias significativas entre grupos: $\mathrm{p}<0,05$; prueba U de Mann-Withney o t de Student según corresponda. 
vaca se asocia con mayor sensibilización a su proteína; ${ }^{25}$ se postula que el incorporar tempranamente una fórmula láctea artificial en este grupo de alto riesgo favorecería la aparición de los síntomas en los lactantes. ${ }^{26}$ Por otra parte, los efectos beneficiosos de la lactancia materna han sido históricamente descritos en niños con predisposición genética a alergia. ${ }^{27} \mathrm{~A}$ pesar de ello, el rol protector de la lactancia materna ha sido recientemente cuestionado; ${ }^{28}$ diversos estudios han comunicado asociación entre duración de la lactancia materna con mayor riesgo de desarrollar asma, atopia y dermatitis atópica. ${ }^{29}$ Sin embargo, en una revisión crítica del tema, Muraro y cols..$^{30}$ concluyen que la lactancia materna exclusiva por lo menos por 4 meses en lactantes de alto riesgo de desarrollar enfermedad atópica, se asocia con una menor incidencia acumulativa de desarrollar alergia a la proteína de leche de vaca a los 18 meses de edad.

La sintomatología gastrointestinal y de piel resultó ser la causa más frecuente que llevó a sospechar un trastorno alérgico. Entre las primeras destacan la diarrea, cólico, reflujo gastroesofágico, constipación y deposiciones con sangre, todas manifestaciones frecuentes en el grupo de edad evaluado. Los resultados coinciden con lo comu- nicado por Sicherer, ${ }^{31}$ quien revisó el espectro de las manifestaciones clínicas gastrointestinales alérgicas en lactantes y niños concluyendo que estos síntomas estarían asociados a fenómenos alérgico en las edades tempranas de la vida. ${ }^{31}$

Se podría postular que los síntomas antes descritos son lo "esperado" debido a que son síntomas frecuentes en el primer año de vida, habitualmente explicados por las limitaciones fisiológicas existentes en esas edades; pero conviene recordar que estos pacientes fueron referidos al centro de especialidad debido a la falta de respuesta al tratamiento de esos síntomas, efectuado en el nivel de salud primaria, y ese es justamente uno de los criterios internacionalmente consensuados en la actualidad para sospechar que las manifestaciones puedan ser alérgicas. Por otro lado, la piel es uno de los órganos blanco que más a menudo está involucrado en reacciones de hipersensibilidad, con un amplio espectro de síntomas, como dermatitis, urticaria y angioedema, todas presentes comúnmente en reacciones de alergia alimentaria. ${ }^{32}$ En nuestra muestra, la dermatitis atópica fue mayormente comunicada entre lactantes alérgicos y fue francamente infrecuente en los controles, lo que coincide con lo publicado en la bibliografía.

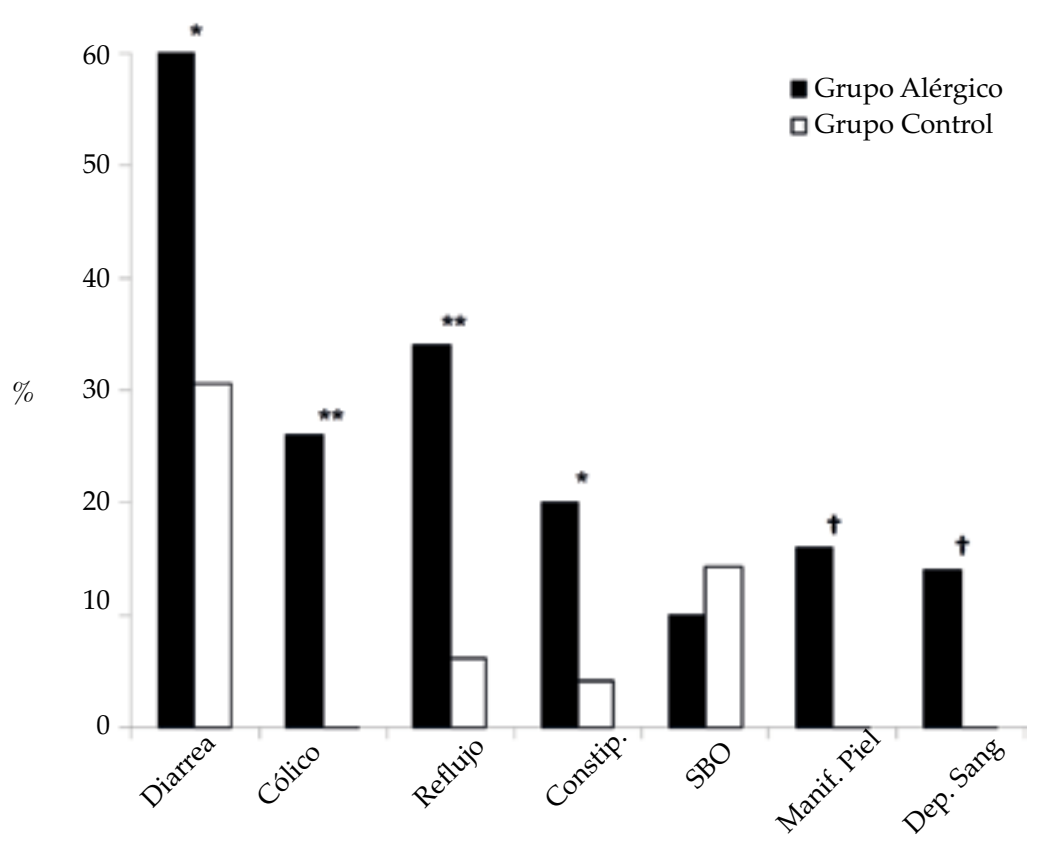

Diferencias significativas entre ambos grupos, prueba ji al cuadrado, ${ }^{*} \mathrm{p}<0,003,{ }^{* *} \mathrm{p}<0,0003,+\mathrm{p}<0,004$.

SBO: síndrome bronquial obstructivo.

Dep. Sang: deposiciones sanguinolentas.

Constip.: constipación.

Manif. Piel: manifestaciones en piel. 
En conjunto, los resultados sugieren que ante síntomas como los descritos, en un niño con historia familiar de alergia, resulta apropiado realizar un diagnóstico presuntivo de alergia y referirlo al especialista. Dada la alta frecuencia de los síntomas aludidos, la identificación del antecedente familiar representaría una herramienta útil para decidir la referencia o mantención del paciente en el consultorio local.

Nuestros resultados muestran, además, que los niños alérgicos presentan diferencias significativas en relación a la talla actual contra el grupo control, datos que pueden ser explicados por el diagnóstico tardío. Este hallazgo es relevante en la vigilancia nutricional que efectúan los programas materno-infantiles, siendo clara la conveniencia de hacer su derivación y diagnóstico lo más precozmente posible, con tratamiento dietoterapéutico especializado.

Es importante tener en cuenta que los sujetos controles se reclutaron de distintos centros de la Región Metropolitana, por lo que no fue posible controlar todas las variables ambientales que podrían influir en los resultados del estudio. Otra limitación tiene relación con el sesgo de memoria, ya que fueron las madres quienes describieron el orden cronológico de introducción de alimentos a sus hijos y entregaron el antecedente de la historia familiar. Por otro lado, la principal fortaleza fue la posibilidad de contar con pacientes diagnosticados en un centro de referencia nacional para el diagnóstico clínico de alergia.

\section{CONCLUSIONES}

Este estudio muestra que, en lactantes, la introducción precoz de fórmula láctea se asoció con la presencia de alergia alimentaria y que el factor de riesgo más importante en el desarrollo de ésta es el antecedente familiar de alergia de los padres. Es importante considerar estos resultados al momento de indicar la ingesta de fórmulas lácteas artificiales, especialmente en lactantes cuyos padres tienen antecedentes de alergia.

\section{Agradecimientos}

En forma especial, a los padres de los niños que participaron en el estudio, por el apoyo recibido durante el desarrollo de este trabajo. A las Sras. Adriana Haberle, enfermera; Verónica Martínez, paramédico y Pamela Valenzuela, secretaria de la sección de Inmunología, VIH y Alergias; a la Escuela de Nutrición y Dietética, y al Dr. Hugo Muñoz, todos pertenecientes a la Universidad de Chile.

\section{BIBLIOGRAFÍA}

1. Mayer L. Mucosalimmunity. Pediatrics 2003;111(6Pt3):1595600.

2. Stanley S. Oral tolerance of food. Curr Allergy Asthma Rep 2002;2(1):73-7.

3. Zeiger RS. Food allergen avoidance in the prevention of food allergy in infants and children. Pediatrics 2003;111(6 Pt 3):1662-71.

4. Sicherer SH, Burks AW. Maternal and infant diets for prevention of allergic diseases: understanding menu changes in 2008. J Allergy Clin Immunol 2008;122(1):29-33.

5. Muche-Borowski C, Kopp R, Reese I, Sitter H, et al. Allergy prevention. Dtsch Arztebl Int 2009;106(39):625-31.

6. Prescott SL, Smith P, Tang M, Palmer DJ, et al. The importance of early complementary feeding in the development of oral tolerance: concerns and controversies. Pediatr Allergy Immunol 2008;19(5):375-80.

7. Sampson HA. Adverse reaction to foods. En: Adkinson NF Jr., et al. Middleton's allergy principles and practice, Mosby: Philadelphia; 2007. Págs.1619-44.

8. Brandtzaeg P. Mucosal immunity: integration between mother and the breast-fed infant. Vaccine 2003;21(24):3382-8.

9. Al-Muhsen S, Clarke AE, Kagan RS. Peanut allergy: an overview. CMAJ 2003;168(10):1279-85.

10. Arbes SJ Jr, Gergen PJ, Elliott L, Zeldin DC. Prevalences of positive skin test responses to 10 common allergens in the US population: results from the third National Health and Nutrition Examination Survey. J Allergy Clin Immunol 2005;116(2):377-83.

11. Rona RJ, Keil T, Summers C, Gislason D, et al. The prevalence of food allergy: a meta-analysis. J Allergy Clin Immunol 2007;120(3):638-46.

12. Branum AM, Lukacs SL. Food allergy among U.S. children: trends in prevalence and hospitalizations. NCHS Data Brief 2008;(10): p. 1-8.

13. Grundy J, Matthews S, Bateman B, Dean T, Arshad SH. Rising prevalence of allergy to peanut in children: Data from 2 sequential cohorts. J Allergy Clin Immunol 2002;110(5):784-9.

14. Greer FR, Sicherer SH, Burks AW. Effects of early nutritional interventions on the development of atopic disease in infants and children: the role of maternal dietary restriction, breastfeeding, timing of introduction of complementary foods, and hydrolyzed formulas. Pediatrics 2008;121(1):183-91.

15. Agostoni C, Decsi T, Fewtrell M, Goulet O, et al. Complementary feeding: a commentary by the ESPGHAN Committee on Nutrition. I Pediatr Gastroenterol Nutr 2008;46(1):99-110.

16. Joseph CL, Owby DR, HavstadSL, WoodcroftKL, etal.Early complementary feeding and risk of food sensitization in a birth cohort. J Allergy Clin Immunol 2011;127(5):1203-10 e5.

17. Sausenthaler S, Heinrich J, Koletzko S. Early diet and the risk of allergy: what can we learn from the prospective birth cohort studies GINIplus and LISAplus? Am J Clin Nutr 2011;94(6 Suppl):2012S-2017S.

18. European Society for Opinionand Marketing Research. The ESOMAR Standard Demographic Classification. A System of International Socio-Economic Classification of Respondents to Survey Research; Amsterdam; 1997.

19. WHO Multicentre Growth Reference Study Group. WHO Child Growth Standards: Growth velocity based on weight, length and head circumference: Methods and development. Geneva: World Health Organization: 2009; Pág. 242.

20. Kahn A, Rebuffat E, Blum D, Casimir G, et al. Difficulty in initiating and maintaining sleep associated with cow's milk allergy in infants. Sleep 1987;10(2):116-21.

21. Pizarro T. Guía de alimentación del niño menor de dos años. Departamento de nutrición y ciclo vital, división de 
prevención y control de enfermedades. Santiago: Ministerio de Salud; 2005; Págs. 7-29.

22. Calvo MC. Regresión logística no condicionada y tamaño de muestra: una revisión bibliográfica. Rev Esp Salud Pub 2002;76:85-93.

23. Cogswell JJ. Influence of maternal atopy on atopy in the offspring. Clin Exp Allergy 2000;30(1):1-3.

24. London SJ, James Gauderman W, Avol E, Rappaport EB, Peters JM. Family history and the risk of early-onset persistent, early-onset transient, and late-onset asthma. Epidemiology 2001;12(5):577-83.

25. Host A. Importance of the first meal on the development of cow's milk allergy and intolerance. Allergy Proc 1991;12(4):227-32.

26. Björkstén B. Does breast-feeding prevent the development of allergy? Immunol Today 1983;4:215-17

27. Kneepkens CM, Brand PL. Clinical practice: Breastfeeding and the prevention of allergy. Eur J Pediatr 2010;169(8):911-7.
28. Ludvigsson JF, Mostrom M, Ludvigsson J, Duchen K. Exclusive breastfeeding and risk of atopic dermatitis in some 8300 infants. Pediatr Allergy Immunol 2005;16(3):201-8.

29. Benn CS, Wohlfahrt J, Aaby P, Westergaard T, et al. Breastfeeding and risk of atopic dermatitis, by parental history of allergy, during the first 18 months of life. Am J Epidemiol 2004;160(3):217-23.

30. Un Muraro A, Dreborg S, Halken S, Host A, et al. Dietary prevention of allergic diseases in infants and small children. Part III: Critical review of published peer-reviewed observational and interventional studies and final recommendations. Pediatr Allergy Immunol 2004;15(4):291-307.

31. Sicherer SH. Clinical aspects of gastrointestinal food allergy in childhood. Pediatrics 2003;111(6 Pt 3):1609-16.

32. Burks W. Skin manifestations of food allergy. Pediatrics 2003;111(6 Pt 3):1617-24.

En Japón los únicos ciudadanos que no están obligados

a inclinarse en reverencia frente al emperador son los educadores.

La razón es que los japoneses sostienen que sin educadores no podría haber emperadores. 